\section{Was hat PISA 2000 den Mathematikerinnen und Mathematikern zu sagen?}

\author{
von Kristina Reiss und Günter Törner
}

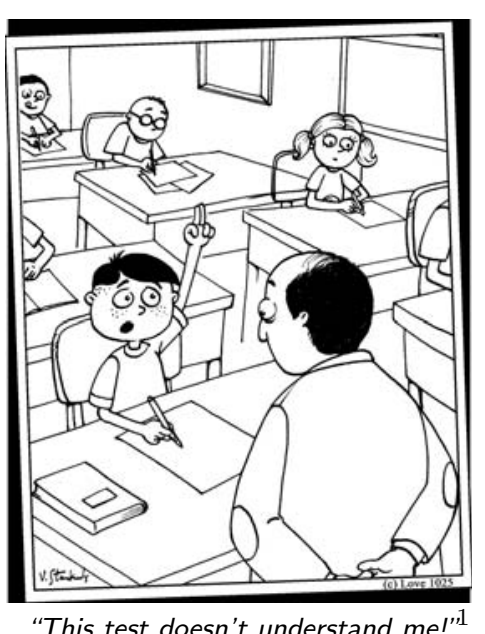

"This test doesn't understand me!"11

Seit dem 4. Dezember 2001 liegen die ersten Ergebnisse der PISA-Studie ${ }^{2}$ (Programme for International Student Assessment) vor. In dieser Studie, an der 32 Länder innerhalb und außerhalb der OECD beteiligt waren, werden die Lesekompetenz, die mathematische Grundbildung und die naturwissenschaftliche Grundbildung in der Altersgruppe der 15-Jährigen betrachtet. Zusätzlich wurden Daten erhoben, die individuelle Merkmale, das familiäre Umfeld und schulbezogene Aspekte betreffen. Mehr als 50000 Schülerinnen und Schüler nahmen in der Bundesrepublik an der ersten Untersuchung, PISA 2000 genannt, teil. Das wenig erfreuliche, aber leider nicht unerwartete Abschneiden der deutschen Jugendlichen in den Leistungstests hat PISA nach TIMSS einen erheblichen Stellenwert in der bildungspolitischen Diskussion gegeben. Es ist zu erwarten, dass wir dieses Schlagwort noch geraume Zeit vernehmen werden.

Wir hatten in früheren Heften bereits auf die anstehenden Erhebungen und möglicherweise ernüchternden Ergebnisse hingewiesen. Die Deutsche Mathematiker-Vereinigung ${ }^{3}$ wie auch die Gesellschaft für Didaktik der Mathematik $(\mathrm{GDM})^{4}$ haben sofort mit Presseerklärungen reagiert, in denen auf dringend notwendig scheinende Konsequenzen hingewiesen wurde. Zusätzlich hat der seinerzeitige Präsident Prof. Dr. Gernot Stroth einen offenen Brief an die zum Zeitpunkt der Veröffentlichung amtierende KMK-Vorsitzende, Frau Dr. Annette Schavan, wie auch an die Ministerin für Bildung und Forschung, Frau Edelgard Bulmahn, gesandt.

Doch die Auseinandersetzung mit PISA und den möglichen Folgen hat gerade erst begonnen. Auch in den nächsten Jahren werden wir in dieser Hinsicht mit eher unbequemen Botschaften rechnen müssen. Wir möchten daher eine Diskussion darüber beginnen, inwieweit wir als Mathematikerinnen und Mathematiker Einfluss auf die Bearbeitung unstrittiger Defizite nehmen können und sollen. Sicherlich sind wir nicht die Einzigen, die sich mit diesen Fragen auseinander setzen müssen. PISA hat an erster Stelle auch deutlich gemacht, dass nicht nur fachbezogene Aspekte eine Rolle bei der Erklärung von Leistungs- varianz spielen. So macht es nachdenklich, dass die deutschen Schülerinnen und Schüler in der Länderrangskala sowohl im Hinblick auf die Mathematikleistungen als auch im Hinblick auf die Lesekompetenz nur einen Platz im unteren Mittelfeld erreichen. Genauso macht es nachdenklich, dass Schulleistung auch zu Beginn des 3. Jahrtausends in unserem Land noch immer eng mit der sozialen Herkunft korreliert. Es liegt auf der Hand, dass die Ursachen nicht monokausal erklärt werden können, sondern wohl aus einer Mischung aus wenig günstigen Bedingungen bestehen, zu denen neben allgemein gesellschaftlichen auch schulische und hier wiederum auch auf das Fach bezogene Aspekte beitragen.

Es ist natürlich leicht, mit einseitigen Schuldzuweisungen zu reagieren. So konnte man in den letzten Monaten vielfach in der Presse lesen, wo die angeblichen Ursachen im Bildungssystem zu suchen seien. Ob man nun dem Unterricht in der Grundschule oder den kaum vorhandenen Ganztagesschulen Erklärungsbedeutung zumessen will, macht keinen Unterschied. Zum jetzigen Zeitpunkt sind solche Folgerungen nicht möglich. Man muss deutlich sehen, dass PISA Defizite beschrieben und Zusammenhänge offen gelegt hat, die Ursachen aber aus der Studie

\footnotetext{
1 Mit freundlicher Genehmigung von Jason Love, http://www.jasonlove.com

2 http://www.mpib-berlin.mpg.de/PISA/

3 http://www.mathematik. uni-bielefeld.de/DMV/archiv/memoranda/pisa01.html

4 http://www.max04.mathematik.uni-wuerzburg.de/ gdm/aktuelles/pisa_gdm.html
} 
nicht vorschnell abgeleitet werden können. Insofern wollen wir uns auch keineswegs an einer spekulativen bildungspolitischen Diskussion beteiligen. Wir fragen uns lediglich, welche der im Rahmen von PISA als relevant für die Mathematikleistung betrachteten Aspekte wir als Mathematiker und Mathematikerinnen an den Universitäten in fachlicher und fachdidaktischer Perspektive mittelbar und unmittelbar beeinflussen können.

Dieser Artikel soll eine Diskussion anstoßen, für die wir uns vor allem Sachlichkeit wünschen. Gestatten Sie dennoch eine provokative Frage vorweg. Wann haben Sie, lieber Leser, liebe Leserin, zuletzt an einem ganz normalen, alltäglichen Mathematikunterricht in irgendeiner Schule teilgenommen? Wenn Ihre Erfahrungen mit Unterricht ein paar Jahre zurück liegen sollten, dann nehmen Sie doch PISA zum Anlass, einen aktuellen Einblick in die Schulwirklichkeit zu finden und Wissen über Unterricht aus „erster Hand" aufzubauen. Immer wieder wird in Diskussionen deutlich, dass vermeintliches Wissen über Unterricht nicht auf eigenen Erfahrungen, sondern auf Gehörtem und Gelesenem basiert. Wir denken, dass auch indirekte Erfahrungen, wie sie Hochschullehrer etwa über die Beurteilung der mathematischen Kompetenz ihrer Studienanfänger machen oder wie ein jeder von uns sie im privaten Umfeld in der Rolle als Mutter, Vater, Onkel, Tante macht (ganz unabhängig davon, ob es gute oder schlechte Erfahrungen sind) keinesfalls den direkten Kontakt zum Lernort Schule ersetzen können. Eigene Erfahrungen ändern zwar nichts an den wesentlichen Erkenntnissen aus PISA, sie lassen aber eine eigene Einordnung zu und können so vielleicht zu der oben gewünschten Sachlichkeit der Diskussion beitragen.

Die folgenden Abschnitte sollen einige basic facts zu PISA liefern und haben die Funktion einer Diskussionsgrundlage, die kurz und knapp in die Thematik einführen soll. Dabei können nicht alle wesentlichen Aspekte von PISA beschrieben werden. So müssen wir beispielsweise darauf verzichten, etwas zum bedrückenden Zusammenhang zwischen Schulleistung und sozialer Herkunft zu sagen. Wir halten diesen Zusammenhang für eines der Ergebnisse von PISA, um die sich unsere Gesellschaft dringend kümmern muss und die Konsequenzen fordern. Für die vertiefte Lektüre seien deshalb weitere Texte empfohlen, auf die wir uns hier auszugsweise beziehen: das internationale Rahmenkonzept von PISA [5], das Rahmenkonzept des Deutschen PISA-Konsortiums [4], die ersten deutschen PISA-Ergebnisse 2000 [3], veröffentlicht durch das Deutsche PISAKonsortium, die offizielle Stellungnahme der OECD zu den Ergebnissen [6] und nochmals zur Erinnerung die deskriptiven deutschen TIMSS/II-Ergebnisse [1] sowie die sehr empfehlenswerten Auswertungen der deutschen TIMSS/III-Erhebungen [2]. Darüber hinaus hat das Bundesministerium für Bildung und Forschung erst kürzlich eine Broschüre herausgegeben, in der die Ergebnisse von TIMSS die Grundlage für Impulse an Schule und Unterricht bilden (vgl. [7]).

\section{Rahmendaten von PISA}

\subsection{Was misst PISA?}

Es waren nicht zuletzt die wenig erfreulichen TIMSSErgebnisse in einigen OECD-Staaten, die eine gründlichere Bestandsaufnahme der Bildungs- und insbesondere Mathematikleistungen nahe gelegt haben. Das Grundkonzept von PISA besteht darin, dass schulische Leistungen im Spiegel mehrerer Kompetenzbereiche erfasst und beurteilt werden sollten. So wurden die Kompetenzbereiche Lesekompetenz ( reading literacy), mathematische Grundbildung (mathematical literacy) und naturwissenschaftliche Grundbildung (scientific literacy) zu einem Bündel von Schlüsselqualifikationen geschnürt. Daneben geht es in der Untersuchung um fachübergreifende Kompetenzen (cross-curricular competencies) wie beispielsweise die Fähigkeit zum selbstregulierten Lernen oder die Fähigkeit zur Kooperation und Kommunikation, wobei auch mögliche Zusammenhänge zwischen diesen Faktoren untersucht werden.

Wenn wir also von den PISA-Ergebnissen reden, so geht es zunächst einmal um ein ganzes Konzept, das in den Erhebungen PISA 2000, PISA 2003 und PISA 2006 realisiert wird. Die Erhebungen haben einen jeweils anderen Schwerpunkt, der in den Hauptbereichen Lesekompetenz (2000), Mathematik (2003) und Naturwissenschaften (2006) liegen wird. Die untersuchte Stichprobe besteht aus 15-jährigen Schülerinnen und Schüler. Es handelt sich somit um eine Altersgruppe, die in fast allen OECD-Mitgliedsstaaten noch der Vollzeitschulpflicht unterliegt. Während sich die Schülerinnen und Schüler in zwei Dritteln der Testzeit einem Hauptbereich widmen, verteilt sich die restliche Zeit auf alle anderen Bereiche, wobei wiederum in den verschiedenen Erhebungen unterschiedliche Schwerpunkte gebildet werden. Auf diese Weise können zusammenfassende Leistungsprofile erfasst werden. Mit anderen Worten: Wir registrieren und diskutieren an dieser Stelle erste internationale wie auch nationale Ergebnisse der Erhebung des Jahres 2000, in denen mathematische Grundbildung zwar eine Rolle spielte, müssen aber beachten, dass sich die Untersuchung erst im Jahr 2003 schwerpunktmäßig mit Mathematik befassen wird. 


\subsection{Mathematische Literalität als Grundbildungsansatz}

Während bei TIMSS die Testaufgaben mit Blick auf die Lehrpläne entwickelt wurden (die Curriculumvalidität von TIMSS/II betrug für das deutsche Sample 95 Prozent), liegt der besondere Charakter des mathematischen PISA-Testprogramms in der noch stärkeren Ausrichtung an einer mathematischen Grundbildung unter deutlicher Berücksichtigung fachdidaktischer Aspekte. Doch wer setzt die Norm, wessen "Philosophie" von mathematischer Bildung war für die Auswahl der Testitems maßgebend?

Basis ist die so genannte mathematical literacy, unter der man die Fähigkeit versteht, die Rolle, die Mathematik in der Welt spielt, zu erkennen und zu verstehen, begründete mathematische Urteile abzugeben und sich auf eine Weise mit der Mathematik zu befassen, die den Anforderungen des gegenwärtigen und künftigen Lebens einer Person als eines konstruktiven, engagierten und reflektierenden Bürgers entspricht ([5], S. 41). Mathematische Kompetenz besteht also im Rahmen von PISA nicht nur aus der Kenntnis mathematischer Sätze und Regeln und der Beherrschung mathematischer Verfahren. Mathematische Kompetenz zeigt sich vielmehr im verständnisorientierten Umgang mit Mathematik und in der Fähigkeit, mathematische Begriffe als „Werkzeuge“ in einer Vielfalt von Kontexten einzusetzen. Mathematik wird als wesentlicher Inhalt unserer Kultur angesehen, gewissermaßen als eine Art Sprache, die von den Schülerinnen und Schülern verstanden und funktional genutzt werden sollte.

Es kann nicht von der Hand gewiesen werden, dass dieser Begriff der mathematischen Grundbildung in der angelsächsischen bildungstheoretischen Diskussion stärker als bei uns in der Schulwirklichkeit verankert ist. Das belegen beispielsweise die Empfehlungen des National Council of Teachers of Mathematics (NCTM) ${ }^{5,6}$ in den Vereinigten Staaten zum Mathematikunterricht. Hier sind fachbezogene und fachübergreifende Principles und Standards die Grundlage, weniger die mathematischen Inhaltsbereiche. Bei Didaktikern ist dieser Ansatz aber auch in der Bundesrepublik gut bekannt, ${ }^{7}$ realisiert er doch Ideen des in die Niederlande emigrierten deut- schen Mathematikers Hans Freudenthal. ${ }^{8}$ Freudenthals Sichtweise findet dabei eine hohe Zustimmung, die Realisierung steckt allerdings noch in den Kinderschuhen. Die niederländische Konzeption der Realistic Mathematics Education (RME) ist als konkrete Umsetzung dieser Ideen in der Schulwirklichkeit anzusehen. Um missverständlichen Fehlinterpretationen hinsichtlich des Wortes realistic vorzubeugen, zitieren wir aus de Lange $^{\text {9: }}$

Eine realistische Problemstellung (real world problem) wird benutzt, um mathematische Konzepte zu entwickeln. Dieser Prozess kann begriffliche Mathematisierung genannt werden: Es geht nicht in erster Linie darum, die Problemstellung zu lösen, um Problemlösefähigkeiten zu entwickeln, sondern die entscheidende Bedeutung liegt in der damit ermöglichten Erkundung neuer mathematischer Begriffe.

Mit anderen Worten: Es geht wesentlich um die Aneignung mathematischer Begrifflichkeiten in einem authentischen Kontext. Dabei unterstellt PISA, dass die konkrete Bearbeitung und Lösung einer mathematischen Aufgabenstellung als Prozess der Erstellung, Verarbeitung und Interpretation eines mathematischen Modells verstanden werden kann. In diesem Sinn lassen sich die Aufgaben des PISA-Tests nach zwei Arten der Modellierung klassifizieren: rechnerische und begriffliche Modellierungsaufgaben.

\subsection{Mathematische Grundbildung - Anspruch und Wirklichkeit}

Die Orientierung im Sinne einer mathematical literacy wird auch in der in Deutschland geführten Diskussion immer wieder sichtbar. Die Autoren des deutschen PISA-Berichts (vgl. [3]) verweisen insbesondere auf die von Heinrich Winter ${ }^{10}$ formulierten Aspekte. Stärker als im Konzept der Realistic Mathematics Education wird in Winters Ansatz hervorgehoben, dass zur mathematischen Grundbildung auch gehört, Mathematik als „deduktiv geordnete Welt eigener Art" zu sehen. Gerade der deutsche Mathematikunterricht orientiert sich vor dem Hintergrund eines solchen Rahmens stark an mathematischen Inhaltsbereichen, also an „Stoffgebieten“, eine Sichtweise, die bei der mathematical literacy im Sinne von PISA letztlich weniger relevant ist. Entsprechend

\footnotetext{
5 National Council of Teachers of Mathematics (NCTM). 1991. Professional standards for teaching mathematics. Reston, VA: National Council of Teachers of Mathematics.

6 National Council of Teachers of Mathematics (NCTM). 2000. Principles and standards for school mathematics. Reston, VA: National Council of Teachers of Mathematics.

7 Hier wird das Problem deutlich, wie zurückhaltend der Einfluss wissenschaftlicher Positionen auf die Bildungsadministration und die Lehrplangestaltung ist.

8 Freudenthal, H. 1973. Mathematik als pädagogische Aufgabe I/II. Stuttgart: Klett.

9 De Lange, J. 1996. Real problems with real world mathematics. In C. Alsina, J.M., Alvarez, M., Niss, A., Pérez, L. Rico et A. Sfard (Eds.), Proceedings of the 8th International Congress on Mathematical Education. pp. 83-110. Sevilla: S.A.E.M. Thales.

10 Winter, H.. 1995. Mathematikunterricht und Allgemeinbildung. Mitteilungen der Gesellschaft für Mathematik 61, 37-46.
} 
den Vereinbarungen bei der Entwicklung der PISATests können solche Aufgabenstellungen aber im nationalen Ergänzungskonzept berücksichtigt werden.

Den Kernaspekten einer mathematischen Grundbildung (rechnerische Modellierungsaufgaben, begriffliche Modellierungsaufgaben) sind insgesamt 31 so genannte internationale PISA-Aufgaben zuzuordnen; daneben gibt es weitere 86 so genannte nationale PISA-Aufgaben. Es wurden vermehrt innermathematische Fragestellungen aufgenommen und auch ,technische Aufgaben", die nur Fertigkeiten und Faktenwissen erfordern. Zusätzlich wurde die nationale Ergänzung dazu benutzt, die herkömmlichen Stoffgebiete (z. B. Arithmetik, Algebra, Geometrie) ausgewogen im Test abzubilden ([3], Seite 156). Der Vollständigkeit halber sollte erwähnt werden, dass die sogenannte latente Korrelation zwischen nationalen und internationalen Aufgaben einen Wert um 0,90 erreicht. Dieser Wert, der selbst für eine latente Korrelation außerordentlich hoch ist, macht deutlich, welch engen Zusammenhang national und international verwendete Testitems zeigen.

\section{Ergebnisse}

\subsection{Mathematische Grundbildung im internationa- len Vergleich}

In der Öffentlichkeit fand primär die Rangliste der teilnehmenden Länder Beachtung. Dabei ist die Bundesrepublik Deutschland bei 31 Teilnehmerstaaten und einem geeichten OECD-Durchschnitt des Mittelwertes von 500 mit 490 Punkten im unteren Mittelfeld zu finden. Deutschland befindet sich damit direkt hinter den Vereinigten Staaten, was mit den Ergebnissen aus TIMSS korrespondiert.

Der Standardmessfehler, der angibt, wie genau ein Wert gemessen werden kann, wird mit nur 2,5 angegeben. Damit haben die Ergebnisse einen akzeptablen Grad an Zuverlässigkeit.

Auffallend ist, dass sich die Staaten relativ klar nach Regionen bzw. (im Fall der angelsächsischen Spitzengruppe) nach kulturellen Traditionen gruppieren. Umso bemerkenswerter ist es, dass Deutschland mit einem Mittelwert von 490 deutlich unter den Werten aller übrigen west- und nordeuropäischen Länder, mit Ausnahme von Luxemburg, bleibt (vgl. [3, Seite 175]).

\subsection{Die PISA-Kompetenzstufen}

Die Ergebnisse werden noch aussagekräftiger und besser interpretierbar, wenn man die Zuordnung der Items zu verschiedenen Stufen mathematischer Kompetenz berücksichtigt. Dabei sind insgesamt fünf Kompetenzstufen zu unterscheiden ([3], Seite 159f.).

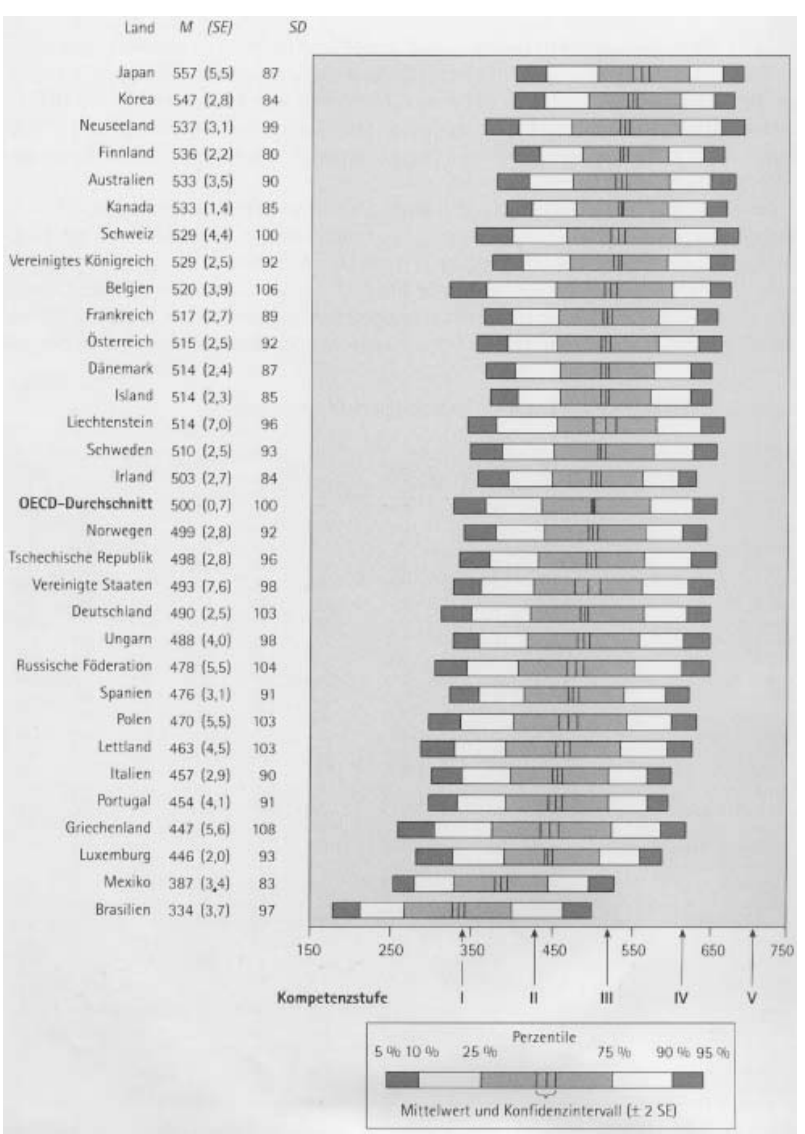

Abbildung 1. Testleistungen der Schülerinnen und Schüler in den Teilnehmerstaaten: Mathematik

Kompetenzstufe I wird über das Rechnen auf Grundschulniveau bestimmt. Immerhin kommen 17 Prozent der deutschen Stichprobe nur auf dieses Niveau und können allerhöchstens Aufgaben bearbeiten, in denen einfaches arithmetisches (oder geometrisches) Wissen in Standardsituationen angewendet wird. Auf Kompetenzstufe II können einfachste begriffliche Modellierungen vorgenommen werden, die in einen außermathematischen Kontext eingebunden sind. Dabei ist auch hier nur dann eine Lösung möglich, wenn eine gewisse Struktur bereits in der Aufgabenstellung vorgegeben ist. Fast ein Drittel der deutschen Probanden, nämlich 32 Prozent, erreichen gerade dieses Niveau. Kompetenzstufe III lässt sich dadurch beschreiben, dass die Fähigkeit zum Modellieren und begriffliches Verstehen auf dem Niveau der Sekundarstufe I gegeben ist. Damit ist vor allem die Beherrschung des Standardschulstoffs in Bezug auf alle Schulformen gemeint. Auch hier ist es ein knappes Drittel der Stichprobe, ganz genau 31 Prozent der Befragten, deren mathematische Fähigkeiten bis zu diesem Bereich gehen. Ein höheres Niveau erreichen schließlich nur wenige deutsche Schülerinnen und Schüler. Aufgaben der Kompetenzstufe IV, die durch das Bewältigen von umfangreichen Modellierungen auf der Basis anspruchsvoller Begriffe gekennzeichnet ist, werden 
von lediglich 12 Prozent der deutschen 15-Jährigen gelöst. Auf der Kompetenzstufe V, die für die komplexe Modellierung und innermathematisches Argumentieren steht, findet man dann gerade noch 1,3 Prozent der befragten Schüler und Schülerinnen in der Bundesrepublik. Sie stellen eine außerst kleine Spitzengruppe dar.

\subsection{Die deutschen Risikogruppen im internationa- len Vergleich}

Betrachtet man die Kompetenzstufen noch einmal genauer, so ist anzumerken, dass immerhin 7 Prozent der deutschen 15-jährigen Schüler noch nicht einmal das Niveau der Kompetenzstufe I erreichen. Damit kommt insgesamt fast ein Viertel nicht über ein höchstens als elementar einzustufendes Wissen hinaus. Im Rahmen von PISA werden diese Jugendlichen als Risikogruppen bezeichnet. Ein weiteres Drittel erreicht nicht das Standardniveau der Sekundarstufe I, d. h. noch nicht einmal die Hälfte der Jugendlichen verfügt über eine den Lehrplanzielen entsprechende mathematische Grundbildung. Die folgenden Graphiken, die sich auf einige ausgewählte Industrienationen beschränken, verdeutlichen das noch einmal.

Man sieht, dass Deutschland und die USA mit einem Anteil von wenig mehr als 40 Prozent an Schülerinnen und Schüler, die den Grundbildungsstandard erfüllen, am Ende dieser Rangliste liegen (vgl. 3.9). Umgekehrt kommt Deutschland eine traurige Spitzenposition hinsichtlich des Anteils der Risikogruppen (Stufe I und darunter) unter den erwähnten Ländern zu, wobei auch hier die USA dicht folgen.

Dieses Bild wird vervollständigt, wenn man sich die Teilnahmequote an der Spitzengruppe (Kompetenzstufe V) vor Augen führt. Lediglich 1,3 Prozent der deutschen Schülerinnen und Schüler sind hier einzuordnen, während im Vereinigten Königreich fast 3 Prozent und in der Schweiz sogar mehr als 3 Prozent dieser Gruppe zuzuordnen sind.

Diese Ergebnisse können nur noch als dramatisch bezeichnet werden und verdienen es, noch einmal herausgehoben zu werden: Nicht einmal die Hälfte der Schülerinnen und Schüler kann Aufgaben, die zum curricularen Standard gehören, mit ausreichender Sicherheit lösen. Fast ein Viertel der 15-Jährigen muss als Risikogruppe eingestuft werden, deren mathematische Grundbildung nur bedingt für die erfolgreiche Bewältigung einer Berufsausbildung ausreicht. (vgl. ([3], Seite 170)).

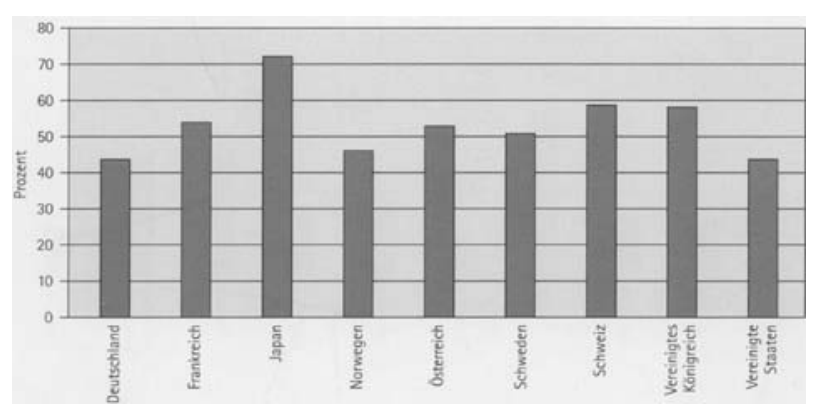

Abbildung 2. Anteil der Schüler, die den Grundbildungsstandard erfüllen

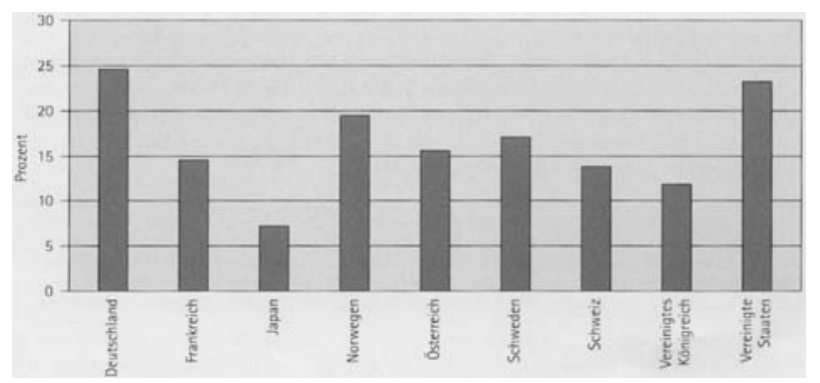

Abbildung 3. Anteil der Risikogruppen

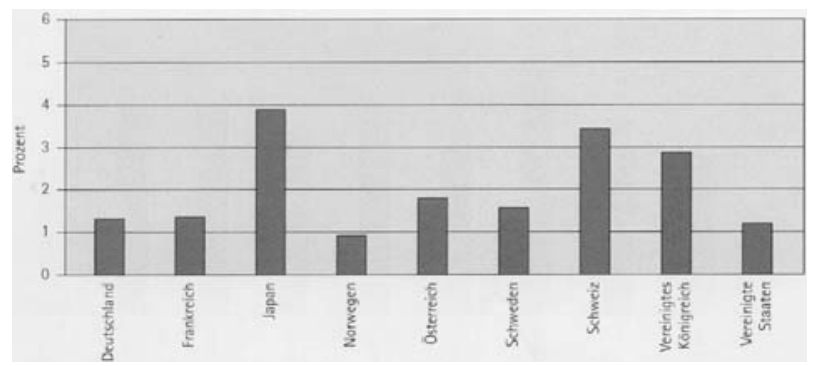

Abbildung 4. Anteil der Spitzengruppe

\section{Konsequenzen}

PISA und die Ergebnisse der Studie werfen die Frage auf, was in der Folge getan werden kann und soll. Wir alle wissen, dass die Konsequenzen nur in einem großen Verbund von Schulen, Hochschulen und der Bildungsadministration gezogen werden können. Alle diese Institutionen müssen ihre Verantwortung erkennen und ihren Teil zur Steigerung der Qualität des Mathematikunterrichts beitragen. Dennoch ist es wichtig, in den einzelnen Communities breit und offen zu diskutieren, welche Konsequenzen sinnvoll und möglich sind und welche Beiträge von anderer Seite wünschenswert erscheinen. In diesen Kontext möchten wir die nachfolgenden Überlegungen eingebettet sehen.

Natürlich lassen sich mit einfachen Erklärungen vortrefflich Schlagzeilen füllen, doch werden sie der Realität nicht gerecht. Im Grunde zeigt jede empirische Untersuchung in Schulen, dass Schulleistungen 
durch ein Gefüge von vielen miteinander verbundenen Variablen bestimmt werden. Umgekehrt kann es aber kein Ziel sein, möglichst viele Gründe zu identifizieren und als Ursachen zu benennen, denn das würde die schulische Mathematikwelt ebensowenig verändern. Dennoch gibt es einige Aspekte, die immer wieder in der Diskussion um bessere Leistungen im Mathematikunterricht und damit schließlich um die Qualitätsentwicklung im Unterricht genannt werden und für die es durchaus empirische Evidenz gibt.

So verweist Jürgen Baumert, unter dessen Leistung am Max-Planck-Institut für Bildungsforschung in Berlin die PISA-Studie 2000 in Deutschland durchgeführt wurde, immer wieder auf die vielen ,überlernten, lange mitgeschleppten" und nur bedingt wirksamen deutschen Unterrichtsskripte, durch die Schüler nicht zu Selbstständigkeit und Neugier animiert würden. Dies wird in einem Beitrag von Eckhard Klieme, Gundel Schümer und Steffen Knoll in der bereits erwähnten Broschüre des BMBF zusammengefasst (vgl. [8]). Typisch für den deutschen Mathematikunterricht ist eine fragend-entwickelnde, lehrerzentrierte Gesprächsführung. In der Regel kennt nur der Lehrer das Ziel des Gesprächs, an dem die Schüler sich mit eher kleinen Beiträgen beteiligen. Dabei hat der Lehrer zumeist klare Erwartungen an die Redebeiträge seiner Schülerinnen und Schüler, deren eigenes Denken kaum aufgenommen, diskutiert und bewertet wird. Alternative Lösungsvorschläge passen dann auch eher nicht in das Konzept eines solchen Unterrichts. Es ist nicht verwunderlich, wenn diese Unterrichtsform einerseits als fordernd für den Lehrer empfunden wird, denn seine Aufmerksamkeit ist permanent notwendig. Es ist genauso wenig verwunderlich, wenn andererseits ein großer Teil der Schülerinnen und Schüler diese Unterrichtsphasen eher als Ritual empfindet, das vor dem eigentlichen „Rechnen" ertragen werden muss.

Im Gegensatz dazu müsste Unterricht in stärkerem Maß die Schülerinnen und Schüler zur Eigenaktivität anregen. Die genannten Autoren führen die Kognitive Aktivierung als eine Grunddimension der Unterrichtsqualität aus (vgl. [8]). Diese Grunddimension hat nach unserer Ansicht einen klaren Fachbezug und sollte uns als Mathematikerinnen und Mathematiker zum Nachdenken über unser Handeln anregen. Doch auch die anderen genannten Grunddimensionen, nämlich die Unterrichts- und Klassenführung und die Schülerorientierung, sollten vor dem fachlichen Hintergrund bedacht werden.

Nebenbei bemerkt: Basiert die Hochschullehre nicht oft ebenfalls auf vielen Skripten? Damit meinen wir nicht die papiernen Vervielfältigungen. Die Erfahrungen mit unseren Studentinnen und Studenten zeigen ja leider zu oft, dass auch unsere Unterrichtsformen nicht unbedingt den gewünschten Erfolg haben.

Wenn wir über Handlungsfelder für die Hochschule nachdenken, unterstellen wir, dass die Lehramtsausbildung einen prägenden Einfluss auf das mathematische Weltbild von Lehrerinnen und Lehrern ausübt, das wiederum für den Unterricht als ein partieller Einflussparameter gesehen wurden muss. Es gibt Untersuchungen, dass dieser Einfluss leider auch negative Folgen für das spätere Unterrichten nach sich ziehen kann. Auf Seite der Hochschule müssen wir uns eingestehen, dass hier nicht immer ein Bild von Mathematik vermittelt wird, das ein positives Verhältnis zum Fach fördert. Ein Beispiel hierfür ist, dass mathematisch umfangreiches und komplexes Modellieren in der gymnasialen Mathematiklehrerausbildung keineswegs ein Standard ist. Wir sind der Meinung, dass diese Studierenden letztendlich mit den Methoden, die sie selbst weitergeben sollen, nicht hinreichend vertraut gemacht werden. Man mag beteuern, genau das würde ja in den Übungen stattfinden. Doch leider geht dieses Argument vielfach an der Realität vorbei, was auch durch Umfragen unter Studierenden bestätigt wird. Nur an wenigen Universitäten werden Lehramtsstudierende genauso gut betreut wie Studierende, die ein Diplom anstreben, nur an wenigen Universitäten wird in ehrlicher Weise versucht, ihre Erwartungen ernst zu nehmen. Nur wenige Institutionen haben die Herausforderung einer Neustrukturierung der Lehramtsausbildung angenommen. Im Gegensatz dazu haben bis in die jüngste Vergangenheit Fachbereiche selbst forschungsorientierte Didaktikstellen in Mathematikstellen umgewidmet (und diese Entwicklung ist bedauerlicherweise noch immer nicht gestoppt). Dahinter steht die falsche Vorstellung, dass man am besten die Reflexion und Vermittlung von schulischer Mathematik (preiswerten) abgeordneten Lehrern und Lehrerinnen überlassen zu können glaubt.

Während Diplomstudierende bei der Anfertigung ihrer Abschlussarbeit originäres mathematisches Arbeiten und Modellieren kennen lernen, steht die Anfertigung der Abschlussarbeit von Lehramtsstudierenden unter enormem Zeitdruck (oft sind es nicht mehr als 3 oder 4 Monate) und befriedigt zumeist weder die Absolventen noch die Themensteller. Ist es dann verwunderlich, wenn Studentinnen und Studenten die schriftliche Hausarbeit gerade nicht im Fach Mathematik schreiben? Wie eigene empirische Untersuchungen des zweiten Autors zeigen, belegen Studenten mit einem naturwissenschaftlichen Zweitfach mathematische Objekte oft nur über diesen eben nicht primär mathematischen Kontext mit Sinn. Dies wäre zunächst nicht zu kritisieren, wenn die Studierenden die mathematische Perspektive anschließend 
hinzu nehmen würden. Doch dies ist eher nicht der Fall, sodass es bei der (einseitigen) Anwendungsperspektive bleibt. Tall und Vinner (1981) sprechen von concept images ${ }^{11}$ - im Gegensatz zu concept definition - und meinen damit, dass ein Begriff mit sehr verschiedenen Bedeutungsinhalten gefüllt sein kann. Für Lehramtsstudierende möchten wir uns gerade in dieser Beziehung ein große Breite wünschen, die als fachliche Voraussetzung für einen guten und schülerorientierten Unterricht angesehen werden muss. Doch daran mangelt es häufig; eine breite Sinngebung mathematischer Begriffe wird durch die Mathematikausbildung in der Regel nicht umfassend konstituiert.

Ganz im Zusammenhang damit steht wohl auch die manchmal bei Kolleginnen und Kollegen zu beobachtende Überzeugung, dass das Transformationsproblem eigentlich trivial ist. Es ist aber keineswegs trivial, den Vektorraumbegriff den schulischen Belangen zugänglich zu machen, auch wenn wir gerne den Lehramtsstudierenden zugestehen wollen, dass sie ein adäquates Verständnis dieser concept definition aufgebaut haben. Wir geben uns damit zufrieden, dass wir sie fachlich qualifiziert haben, alles andere sei ihre eigene Sache und die der weiteren Lehramtsausbildung. Hier beginnt eigentlich die fachdidaktische Aufgabe, deren Bewältigung wir nicht fördern, wenn wir nur von einem methodischen Problem sprechen und diese Diskussion ausschließlich in die Hand der Schulpraxis legen.

Was können wir aber tun, um Lehrer und Lehrerinnen besser zu qualifizieren? Es ist wohl keine Frage, dass wir ein stärkeres Augenmerk sowohl auf die Lehrerausbildung als auch auf die Lehrerfortbildung legen sollten. Nach unserer Ansicht ist hier ein wesentliches Handlungsfeld für Mathematikerinnen und Mathematiker aufgezeigt. Dabei muss es sicherlich darum gehen, fachliche und fachdidaktische Aspekte angemessen und vor allem ineinandergreifend $\mathrm{zu}$ berücksichtigen. Lehrerfortbildung darf nicht auf das Lernen neuer Vokabeln reduziert werden. Stattdessen gehören die mathematischen Inhalte, ihre Einbettung in mathematikbezogene und anwendungsbezogene Kontexte und die didaktisch-methodische Umsetzung zusammen.

Wie wird es weitergehen? Zunächst einmal mit neuen Ergebnissen. Ende Juni werden die ersten Daten im Bundesländervergleich veröffentlicht, sodass die Diskussion noch einmal belebt werden wird. Wir dürfen allerdings nicht vergessen, dass die Botschaft von PISA nicht nur auf Leistungsvergleiche reduziert werden darf. Vielmehr hat PISA uns die Augen geöffnet und dazu motiviert, ein altes Ziel weiter zu verfolgen. Dieses Ziel ist, den Mathematikunterricht in einer sich verändernden Welt für junge Menschen kognitiv anspruchsvoll und zugleich interessant zu gestalten. Der Wille zur Verbesserung der Unterrichtsqualität ist es, der weiterhin unseren Weg bestimmen sollte.

\section{Literatur}

[1] Baumert, J.; Lehmann, R. u. a. 1997. TIMSS - Mathematisch-naturwissenschaftlicher Unterricht im internationalen Vergleich. Deskriptive Befunde. Opladen: Leske + Budrich.

[2] Baumert, J.; Bos, W.; Lehmann, R. (Hrsg.). 2000. TIMSS/III - Dritte Internationale Mathematik - und Naturwissenschaftsstudie - Mathematische und naturwissenschaftliche Bildung am Ende der Schullaufbahn. Band 1: Mathematische und naturwissenschaftliche Grundbildung am Ende der Pflichtschulzeit. Band 2: Mathematische und physikalische Kompetenzen am Ende der gymnasialen Oberstufe. Opladen: Leske + Budrich.

[3] Baumert, J.; Klieme, E.; Neubrand, M.; Prenzel, M.; Schiefele, U.; Schneider,W.; Stanat, P.; Tillmann, K.-J. \& Weiss, M. (Hrsg.). 2001. PISA 2000: Basiskompetenzen von Schülerinnen und Schülern im internationalen Vergleich. Opladen: Leske + Budrich.

[4] Deutsches PISA-Konsortium. 2000. Schülerleistungen im internationalen Vergleich. Eine neue Rahmenkonzeption für die Erfassung von Wissen und Fähigkeiten. Paris: OECD.

[5] OECD. 1999. Measuring Student Knowledge and Skills. A New Framework for Assessment. Paris: OECD Programme for international student assessment.

[6] OECD. 2001. Lernen für das Leben. Erste Ergebnisse von PISA 2000. Paris: OECD Programme for international student assessment.

[7] Bundesministerium für Bildung und Forschung (Hrsg.). 2001. TIMSS - Impulse für Schule und Unterricht. Bonn: BMBF Publik.

[8] Klieme, E.; Schümer, G.; Knoll, St. 2001. Mathematikunterricht in der Sekundarstufe I: „Aufgabenkultur" und Unterrichtsgestaltung. In: Bundesministerium für Bildung und Forschung (Hrsg.). TIMSS - Impulse für Schule und Unterricht (S. 43-57). Bonn: BMBF Publik.

\author{
Adresse der Autoren \\ Prof. Dr. Kristina Reiss \\ Fachbereich 6 - Mathematik \\ Carl von Ossietzky Universität Oldenburg \\ 26111 Oldenburg \\ kristina.reiss@uni-oldenburg.de
}

Prof. Dr. Günter Törner

Institut für Mathematik, Fakultät 4

Gerhard-Mercator-Universität

Lotharstraße 65

47048 Duisburg

toerner@math.uni-duisburg.de

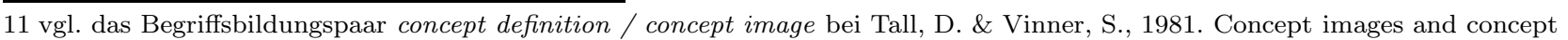
definition in mathematics with particular reference to limits and continuity. Educational Studies in Mathematics $\mathbf{1 2}$, $151-169$. 